\title{
LA GLUCEMIA EN LA ENFERMEDAD DE CARRION Y EN LA BARTONELLOSIS DEL PERRO *
}

Por Julio Pons Muzzo

Las investigaciones de Mann y asociados (1), (2), en el perro hepatectomizado, y los posteriores estudios de Soskin y colaboradores (3), (4), en este mismo animal bajo diferentes condiciones, han demostrado, de modo indudable, que el hígado desempeña un rol primordial en la regulación de la cifra normal de glucosa sanguínea.

Una caída de la glucemia a niveles marcadamente hipoglucémicos, con desaparición del glucógeno hepático y curvas anormales de tolerancia a la glucosa, ha sido constada en animales intoxicados con sustancias químicas diversas (hidrazina, cloroformo, fósforo, dimetilnitrosamina, etc.), que producen necrosis e infiltración grasosa del hígado (5).

En sujetos con enfermedades hepáticas (hepatitis, cirrosis, atrofia amarilla, carcinomatosis, etc.) Conn y asociados (6), Coller y Jackson (7) y otros, han observado sindromes de hipoglucemia espontáneos y curvas de tolerancia anormales.

Considerando que en la Enfermedad de Carrión, aparte de la infiltración grasosa del hígado como alteración casi constante, focos de necrosis centrolobulillares han sido observados en algunos casos (8), (9), (10), y que en la Bartonellosis del perro, los estudios de Weiss (9) y de Munch (11) han demostrado que estos focos necróticos, circundados por una zona de infiltración grasosa, se presentan con frecuencia, creímos de interés determinar la glicemia en ambos procesos.

\footnotetext{
- Estudio realizado en la Sección Quimica Biológica del Instituto Nacional de Higlene y Salubridad.
} 
Esta investigación estaba justificada también desde el punto de vista del síntoma anemia, teniendo en cuenta que varios investigadores han puesto de manifiesto un incremento del azúcar sanguíneo en la Anemia Perniciosa (12), (13) (14).

Hemos estudiado a 8 enfermos carriónicos, en fase hemática y estado anémico, 15 sujetos normales y 25 perros esplenectomizados, infectados con Bartonella Canis.

\section{Sujetos. Animales de experimentación. Métodos.}

Las investigaciones al estado normal fueron efectuadas en sujetos pértenecientes al Ejército, cuya edad variaba entre 20 y 28 años, procedentes en su mayoría de la Sierra pero con un tiempo de permanencia en Lima de 8 meses a 3 años.

Los enfermos carriónicos pertenecian a los hospitales Loayza y Dos de Mayo; el mayor número a este último. En todos la investigación de Bartonellas fué positiva al examen directo y la mayoria presentaban un grado de anemia muy intenso.

De los 25 perros estudiados, 17 eran animales jóvencs, cuyo peso variaba entre 5 y 9 kilos y los restantes tiernos con un peso entre 1 y 3 kilos. Se les hizo previamente una o dos determinaciones al estado normal. luego varias después de la esplenectomia. y una vez infectados los hemos estudiado, en forma periódica, durante todo el desarrollo del proceso. Fueron mantenidos con una dieta standard.

La sangre en todos los casos, humanos y animales, fué tomada en ayunas y se hicieron al mismo tiempo exámenes hematológicos complementarios (numeración de hematies, leucocitos, reticulocitos, grado de parasitismo hepático, reacción de van den Bergh, etc.).

Hemos empleado el micrométodo de Hagedorn Jensen, desproteinizando con zinc según el procedimiento de Somogyi (15), exceptuando a los dos primeros casos humanos y a uros pocos animales, en los cuales usamos el método de Folin Wu. Para evitar la glucolisis durante el transporte de la sangre al laboratorio hemos usado el fluoruro de sodio.

Las determinaciones se realizaron en la sangre total y en el plasma. Las consignadas en el presente trabajo, correspondientes a sujetos normales y enfermos carrionicos, se refieren a la sangre total. Dejamos para uno próximo esperando reunir un inayor número de enfermos - el estudio de la glucosa plasmática y globular.

\section{La glucemia en la Enfermedad de Carrión.}

Como valor medio de glucosa en sangre total, en sujetos adultos normales, hemos obtenido la cifra de $88 \mathrm{mgs} \% \pm 1.05$, con un coeficiente de variación de $6.6 \%$ y variaciones extremas de 78 y 100 mgs\% (Cuadro $\mathrm{N}^{\circ}$ 1.) 
Es tarea difícil hacer un estudio comparativo de los resultados hallados con los que se encuentra en la literatura, principalmente por el hecho que no todas las cifras consignadas representan el verdadero valor de azúcar sanguíneo sino que comprenden además a otras sustancias reductoras (glutation, ergoteonina, etc.). La eliminación de tales sustancias reductoras no fermentables se conseguiría con el método de Folin para sangre "no lacada" (16) o desproteinizando con zinc según Somogyi.

La cifra media hallada por nosotros es ligeramente superior a la obtenida por San Martín (18), empleando el método de Folin para sangre "no lacada" ( $81 \mathrm{mgs} \%$ ).

\section{CUADRO NO 1}

Glucemia normal (sangre total, mgs\%).

\begin{tabular}{c|c|c|c}
\hline $\begin{array}{c}\text { Media } \\
\pm \text { E.P. }\end{array}$ & $\begin{array}{c}\text { Desviación } \\
\text { standard } \\
\pm \mathrm{E} . \mathrm{P} .\end{array}$ & $\begin{array}{c}\text { Coeficiente } \\
\text { de variación } \\
\%\end{array}$ & $\begin{array}{c}\text { Variaciones } \\
\text { extremas }\end{array}$ \\
\hline $88 \pm 1.05$ & $5.82 \pm 1.09$ & 6.6 & $78-100$ \\
\hline
\end{tabular}

Los resultados obtenidos en la Enfermedad de Carrión, los presentamos en el cuadro $\mathrm{N}^{\circ} 2$. En él puede observarse que en la mitad de los enfermos estudiados (casos $2,3,6$ y 8), la cifra de gluccsa en la sangxe total muestra un marcado incremento sobre el nivel normal. La variación en el mismo sentido a que daría lugar la reducción del hematocrito, debido al proceso anémico, quedaría fuera de toda consideración ante la magnitud del aumento constatado. 


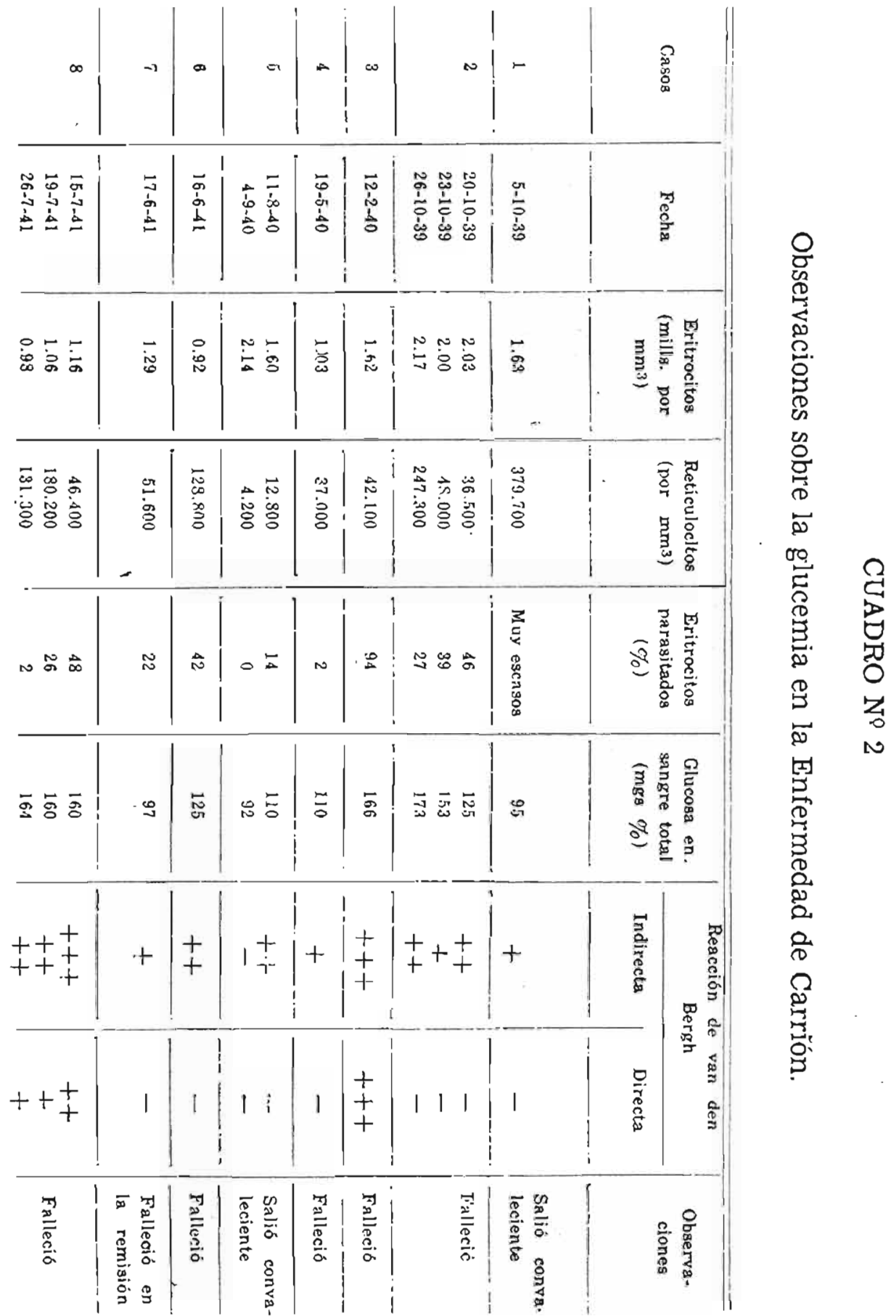




\section{La glucemia en la Bartonellosis del perro *}

En el Cuadro $\mathbb{N}^{\circ} 3$ resumimos, en forma esquemática, el resultado de nuestras observaciones sobre la glucemia en la Bartonellosis experimental del perro. Hemos considerado como alteración en el sentido de una hiperglucemia o hipoglucemia, un incremento o disminución en la cifra de glucosa sanguínea mayor que el $30 \%$ del nivel normal.

De él podemos aeducir, en primer lugar, que en la Bartonellosis del perro ocurren, con cierta frecuencia, variaciones significativas de la glucemia y que éstas pueden ser en más o menos, determinando una huperglucemia o una hipoglucemia.

En seguida, es posible hallar una evidente correlación entre variaciones de la glucemia y severidad del proceso anémico. Con excepción del caso 21 , que falleció a los pocos días de la inoculación, en estado hipoglucémico acentuado, sin dar tiempo a que la anemia se estableciera, los casos que muestran cambios manifiestos en el nivel de azúcar sanguíneo, son aquellos que presentaron anemia de grado severo, la mayoría de los cuales fallecieron espontáneamente.

Por último, observamos que si bien las dos formas de alteración pueden presentarse en un mismo caso, la mayor parte de las veces se constata una sola, siendo ésta la hipoglucemia.

(*) Hacemos hincapie en que este estudio de la glicemia como las determinaciones de glucógeno heoático y las pruebas de tolerancia a la glucosa, que presentamos a continuación, lo hemos realizado preferentemente en animales en desarrollo, teniendo en cuenta que - como ya ha sido señalado (9) - ellos hacen procesos más agudos que los animales adultos.

Despues de la esplenectomia no hemos observado ninguna variacion significativa de la glucemia. 


\section{CUADRO $\mathrm{N}^{\circ} 3$}

\section{Variaciones de la glucemia en la Bartonellosis del perro.}

(Se ha considerado como hiperglucemia o hipoglucemia un incremento o disminución de la glucosa mayor que el $30 \%$ del nivel normal).

\begin{tabular}{|c|c|c|c|c|c|c|}
\hline $\begin{array}{l}\text { No del } \\
\text { perro }\end{array}$ & $\begin{array}{c}\text { Peso } \\
\text { (kilos) }\end{array}$ & $\begin{array}{l}\text { Grado máxi- } \\
\text { mo de ane- } \\
\text { mia (mills. } \\
\text { de eritrocitos } \\
\text { por mm3 }\end{array}$ & $\begin{array}{c}\text { Hidergii- } \\
\text { cemia }\end{array}$ & $\begin{array}{l}\text { Hipogli- } \\
\text { cemia }\end{array}$ & Fallecido & Curado \\
\hline 1 & 1.0 & 0.48 & + & $\cdots$ & $i$. & $\ldots$ \\
\hline 2 & 5.1 & 0.72 & + & + & + & $\cdots$ \\
\hline 3 & 2.0 & 0.57 & -- & + & + & $\cdots$ \\
\hline 4 & 5.2 & 0.60 & - & + & $\cdots$ & + \\
\hline 5 & 1.7 & 0.52 & - & + & + & $\cdots$ \\
\hline 6 & 5.0 & 0.52 & + & + & + & $\cdots$ \\
\hline 7 & 4.1 & 0.72 & - & + & + & $\ldots$ \\
\hline 8 & 4.5 & 1.54 & - & - & $\ldots$ & + \\
\hline 9 & 5.6 & 0.70 & + & - & $\ldots$ & + \\
\hline 10 & 4.0 & 0.50 & - & + & + & $\cdots$ \\
\hline 11 & 1.1 & 0.87 & - & + & \multicolumn{2}{|c|}{ Secrificado } \\
\hline 12 & 5.4 & 2.52 & - & - & $\cdots$ & + \\
\hline 18 & 6.5 & 0.45 & -- & + & + & $\cdots$ \\
\hline 14 & 5.0 & 1.51 & - & - & $\cdots$ & + \\
\hline 15 & 6.6 & 1.89 & - & - & $\cdots$ & + \\
\hline 16 & 1.1 & 0.40 & + & - & \multicolumn{2}{|c|}{ Sacrifleado } \\
\hline 17 & 1.2 & 0.65 & - & + & + & $\cdots$ \\
\hline 18 & 5.3 & 0.70 & + & + & + & $\cdots$ \\
\hline 19 & 0.2 & 1.40 & - & - & $\cdots$ & + \\
\hline 20 & 5.0 & 2.44 & - & - & $\cdots$ & + \\
\hline 21 & 1.2 & 2.25 & - & + & + & $\cdots$ \\
\hline 22 & 2.5 & 0.50 & - & + & \multicolumn{2}{|c|}{ Sacrificado } \\
\hline 23 & 2.8 & 0.93 & - & + & + & $\cdots$ \\
\hline 24 & 2.7 & 0.94 & - & + & + & $\ldots$ \\
\hline 25 & 5.8 & 1.47 & - & - & $\ldots$ & + \\
\hline
\end{tabular}


Realizando ahora un estudio, en forma individual, de los resultados obtenidos, podemos señalar los siguientes hechos de interés :

I-Iniciándose el ascenso o descenso de la glucemia durante el proceso de desglobulización, el estado hiperglucémico o hipoglucémico se presenta en la fase de anemia intensa (figuras $\mathrm{N}^{\circ} 1$ y $\mathrm{N}^{\circ} 2$ ).

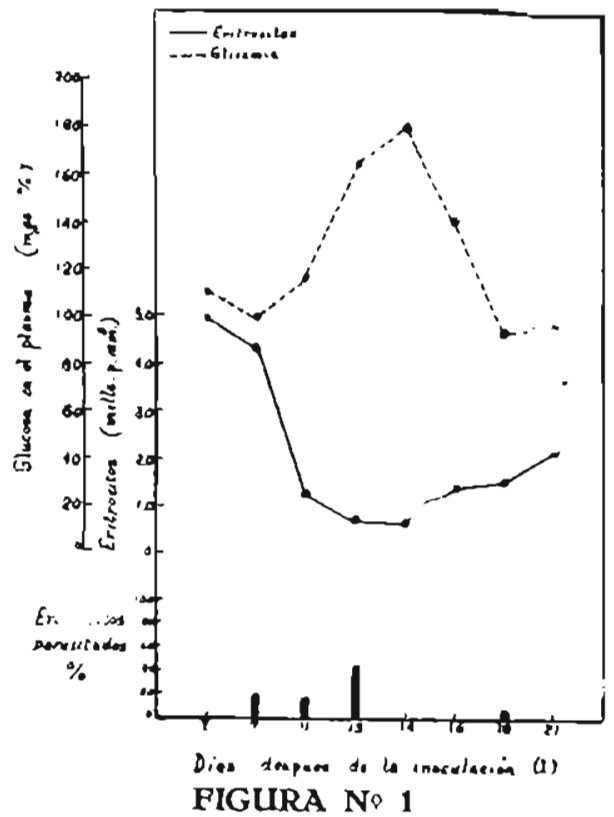

Perro No 9.-Muestra un evidente estado hiperglucémico durante la etapa dx anemia severa, que desaaprece al tener lugar la remisión sanguinea.

II-Cuando en un mismo caso tienen lugar las dos formas de variación de la glucemia, la hiperglucemia precede a la hipoglucemia (figura $\mathrm{N}^{\circ} 3$ ).

III-El marcado descenso de la glucemia sería responsable del estado convulsivo premortal, que nos fué posible constatar en algunos casos. 


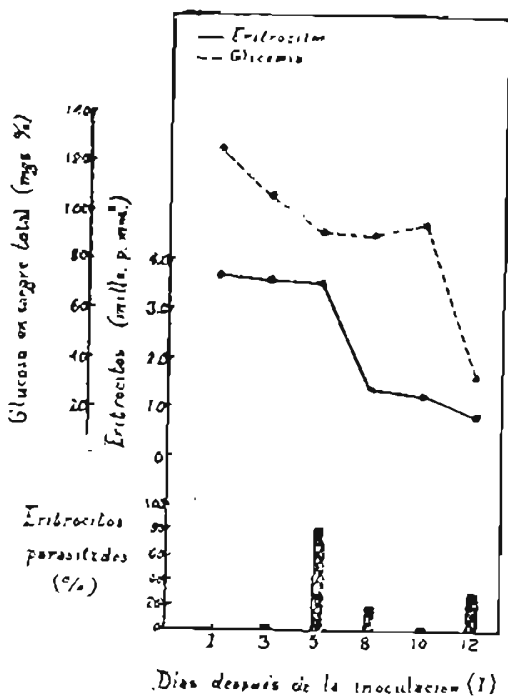

FIGURA No 2

Perro No 24.-Inversamente al perro 9, es una profunda hipoglucemia la que se presenta al final del proceso. Rué un caso muy agudo. Murió en convulsiones.

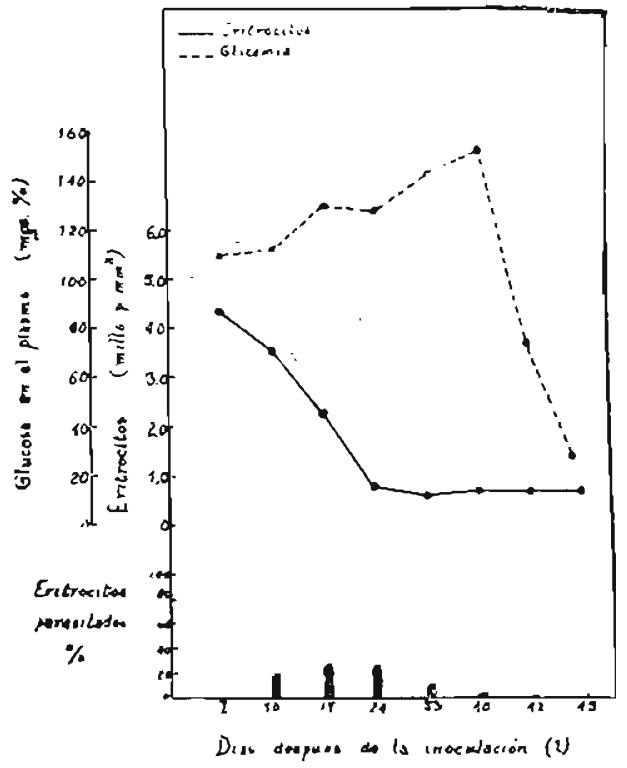

RIGURA No 3

Perro No 8.-Muestra una hipoglucemia terminal precedida de una fase hiperglucémica. 


\section{Discusión.}

Determinando la glucemia en ayunas en sujetos con enfermedad de Carrión, en fase hemática y estado anémico, hemos hallado una hiperglucemia moderada en algunos de ellos. En perros infectados con Bartonella canis, realizando esta investigación en forma más completa, desde la iniciación del proceso hasta la muerte del animal o su curación aparente, hemos constatado también una hiperglucemia en la fase de anemia severa, pero, con más frecuencia, una hipoglucemia en la etapa terminal. Ambas alteraciones han sido observadas en un mismo caso, precediendo la hiperglucemia a la hipoglucemia.

Una hiperglucemia de grado moderado ha sido señalada en los procesos infecciosos febriles, e incluída en el grupo de trastornos producidos por el estado de excitación simpática, que acompaña al período febril de las infecciones agudas (19). Kirstein y Bromberg (20) han constatado esta misma alteración en la fiebre artificial terapéutica. Según ellos, sería el resultado de un cesequilibrio entre un mayor consumo de glucosa a nivel tisular y una sobreproducción de esta sustancia por el hígado.

Los hallazgos de Johnson (12), Kageura (13) y Gittler y St. George (14), de un incremento de la glucemia en la Anemia Perniciosa, cuyo grado sería proporcional a la severidad de la anemia, y aquellos de Kellaway, Gellhorn y Packer (21), de la misma alteración en el gato y conejo sometidos a atmósfera pobre en oxígeno, indicarían que existe alguna relación entre hiperglucemia y anoxemia.

Siendo la Anemia grave de Carrión un proceso de origen microbiano, febril en alto grado, en el que existe también una anoxemia, por la gran reducción de la hemoglobina circular-te (22), la hiperglucemia que hemos constatado puede tener como causa una exagerada glucogenolisis hepática, que sobrepasa las demandas del consumo a nivel de los tejidos, estimulada por el estado infeccioso y quizás también por la anoxia anémica. Tratándose de procesos similares, en igual forma interpretaríamos el incremento de la glucemia observado en la Bartonellosis del perro.

Se comprende que actuando los estímulos necesarios, esta alteración de la glucemia puede no manifestarse, ya por una 
gran utilización de glucosa o por un déficit de glucógeno en el hígado. De esta manera podríamos explicar la escasa frecuencia con que se presenta en la Bartonellosis del perro, en la que, inversamente, la hipoglucemia terminal se constata a menudo.

En algunos perros bartonellósicos fallecidos espontáneamente en anemia severa o hipoglucemia o sacrificados en este estado, hemos constatado - mediante el dosaje químico -un agotamiento de las reservas de glucógeno en el hígado * Habiendo quedado establecido, después de las investigaciones de Mann y asociados (1), (2), en el perro hepatectomizado, y de los posteriores estudios de Soskin (23) y de Houssay (24), sobre la acción de conocidos agentes hiperglucemiantes en este animal en las mismas condiciones, que el hígado es la única fuente de abastecimiento de la glucosa de la sangre, en el organismo en ayunas, este hallazgo explicaría la caída de la glucemia en la etapa final del proceso; pero, se sabe, que el nivel normal de azúcar de la sangre puede mantenerse constante por algún tiempo, después de un período hipoglucémico transitorio, a pesar de haber sido consumidos los depósitos de glucógeno en el hígado - como por ejemplo en el ayuno prolongado -, debido a la neoformación de azúcar a partir de las proteínas y grasas de reserva, principalmente de las primeras. Por consiguiente, una falta de glucógeno y una probable inhibición de la neoglucogenia, ya por escasez de las sustancias básicas necesarias o por una insuficiencia del hígado para realizarla, serían los factores responsables del estado hi-

\footnotetext{
- En cuatro animales fallecidos en hipoglucemia marcada, dos espontáneamente ( $N \circ 13$ y 17) y dos sacrificados, en ayunas, (No 11 y 22), determinamos. inmediatamente despues de la muerte, el contenido de glucógeno del higado. Usamos el método de Sahyun (25). Debido a la cantidad tan pequeña existcate en las muestras de análisis, no nos fué posible hacer una comparación cólo rimétrica exacta en ninguno de los casos. Este fenómeno no ocurrió cuando realizamos la misma investigación en perros convalecientes del proceso bartonellósico.

En un caso sacrificado en hiperglucemia muy acentuada (No 16), el glucógeno hepático estaba tan reducido como en los casos muertos en hipoglucemia.

Sandberg y asociados (26). han demostrado también que la hipoglicemia constatada por ellos en la Bartonellosis de la rata, va acompañada de una desaparición casi total del glucógeno hepático.
} 
poglucémico que se presenta en la etapa terminal de la Bartonellosis grave del perro.

Este gran déficit de glucógeno constatado en la etapa de anemia intensa en la Bartonellosis grave del perro, tendría como primer factor causal, un mayor consumo de glucosa, debido al proceso infeccioso. Parece demostrarlo el resultado de la siguiente experiencia (cuadro $\mathrm{N}^{94}$ ) :

Dos perros tiernos, esplenectomizados, wno al tercer dia de la inoculación con Rartonella canis (A) y el otro no inoculado y libre aparentemente de esta infección (B), son examinados a las 8 a.m., obteniéndose los siguientes resultados principales: perro $A$, eritrocitos 3.44 mills por $\mathrm{mm}^{3}$, eritrocitos parasitados $67 \%$. glucemia $96 \mathrm{mgs}^{2}$; perro B, eritrocitos 4.14 mills. por rnm $^{3}$, eritrocitos parasitados 0 . glucemia $107 \mathrm{mgs} \%$. Luego, son privados de su alimentación ordinaria y a las 4 p.m. se les hace una nueva investigación de la glucemia, correspondiéndole al perro A (infectado) $58 \mathrm{mgs} \%$ y al B (no infectado) $103 \mathrm{mgs} \%$.

\section{CUADRO NP 4}

Variaciones de la glucemia en dos perros en ayuno, uno infectado con Bartonella canis (A) y el otro nó (B).

\begin{tabular}{|c|c|c|c|c|c|}
\hline \multirow{2}{*}{ Perro } & \multirow{2}{*}{$\begin{array}{c}\text { Peso } \\
\text { (kilos) }\end{array}$} & \multirow{2}{*}{$\begin{array}{c}\text { Eritracitas } \\
\text { (mills. por } \\
\text { mms) }\end{array}$} & \multirow{2}{*}{$\begin{array}{c}\text { Eritrocitos } \\
\text { Darasitados } \\
(\%)\end{array}$} & \multicolumn{2}{|c|}{ Glicemia (mag. \%) } \\
\hline & & & & 8 a.m. & 4 p.m. \\
\hline$\underset{\text { (infectado) }}{\mathrm{A}}$ & 2.5 & 3.44 & 67 & 96 & 58 \\
\hline $\begin{array}{c}B \\
\text { (No infec- } \\
\text { tado) }\end{array}$ & 1,9 & 4.14 & 0 & 107 & 103 \\
\hline
\end{tabular}

Aunque ha sido señalado que la correlación entre lesión anatómica hepática y alteración funcional no siempre es evidente (27), (28), debido al gran poder de reserva del hígado - demostrado por Mc Master y Drury (29) en el conejo parcialmente hepatectomizado - y que en las enfermedades hepáticas unas funciones del órgano pueden estar alteradas $\mathrm{y}$ 
otras no (27), (30), (31), el hecho que los perros bartonellósicos que fallecieron en hipoglucemia, en los que nos fué posible realizar el estudio histológico del hígado, * hayan presentado extensas lesiones destructivas e infiltración grasosa, y que algunos de éstos y otros casos en los que se constató la misma variación de la glucemia, exhibieran al mismo tiempo alteraciones en la excreción del pigmento biliar y en otras funciones del hígado, relacionadas con el metabolismo protetico y la coagulación sanguínea, hace pensar que otrc factor determinante del déficit de glucógeno hepático puede ser una insuficiencia de la función glucogénica del hígado.

Varios son los estudios clínicos y experimentales que demuestran la asociación de estado hipoglucémico, lesión del hígado y disminución de la capacidad del mismo para fijar glucógeno. Conn y asociados (6), en pacientes con hepatitis crónica, comprobada en material de biopsia, que mostraban periodos de hipoglucemia espontáneos y en los cuales la calorimetria indirecta indicó una normal oxidación de la glucosa, hallaron una curva de tipo diabético al realizar en ellos la prueba de tolerancia a la glucosa. Iguales resultados obtuvieron Coller y Troost (7) en varias enfermedades del higado (cirrosis, hepatitis sifilítica, colecistitis crónica, hepatitis arsenical, carcinomatosis extensiva). En el campo experimental, Yzume y Lewis (32) observaron una glucogénesis imperfecta, mediante esta misma prueba, en conejos envenenados con la hidrazina - con necrosis central hepática e infiltración grasosa - que presentaron hipoglucemia.

Trabajos experimentales de la pasada década, han demostrado que en la reacción metabólica que determina la curva normal de tolerancia a la glucosa, el hígado juega un papel de primer orden y no el páncreas como se había aceptado, y que la curva diabética de tolerancia se presenta toda vez que la función hepática ha sido suprimida ya total o parcialmente. Soskin, Essex, Herrick y Mann (3) determinando cuantitativamente la entrada y salida de azúcar por el hígado intacto, mediante el thermos tromuhr en perros especialmente preparados, han demostrado que siempre que la tasa sanguí-

- Fué efectuado en el Departamento de Anatomia Patológica de la Facultad de Ciencias Médicas. 
nea de glucosa tiende a elevarse sobre el nivel normal, el hígado responde disminuyendo la producción de glucosa y su salida a la circulación. El estímulo que produce esta resp:lesta inhibitoria sería la glucosa misma de la sangre y el umbral de este estímulo coincide con el nivel normal de glucosa que el animal habitualmente mantiene. La utilización y el almacenaje hacen regresar rápidamente la glucemia a su tasa normal, después de lo cual el hígado reasume su función secretora.

Soskin, Aliweiss y Cohn (33), (34), han mostrado que perros completamente pancreatectomizados, que reciben una constante dosis de insulina, suficiente para mantener un nivel normal de glucemia, producen una curva normal de tolerancia a la glucosa, incluyendo la fase hipoglucémica; pero, perros hepatectomizados con páncreas intacto, recibiendo una constante inyección de glucosa para mantener normal la glicemia, invariablemente producen curvas marcadamente diabéticas.

Coller y Troost (7) han señalado que en el perro, la remoción de grandes bloques de tejido hepático altera la curva de tolerancia en el sentido diabético.

Recientes investigaciones de Curtis y asociados (35) han demostrado que en clínica, la prueba de la glucosa tiene más valor que la de la galactosa, como medida de función hepática. La primera fué positiva en el $90 \%$ de casos de hepatitis mientras que esta última en el $30 \%$.

Realizando esta prueba en cinco animales, los resultados obtenidos nos han demostrado, en forma evidente, una disminución de la capacidad del hígado para fijar glucógeno, en los casos graves, durante la etapa de anemia severa (figura No 4).

La prueba fué realizada al estado normal y después de la inoculación de Bartonella canis. en estado anémico. Mediante una sonda gástrica se les administró 3 gramos de glucosa pura por kilo de peso.

$E_{n}$ tres casos (№ 23 y 24), las curvas obtenidas en el periodo anémico fueron de tipo marcadamente diabético, presentando uno de ellos - el único en que nos fué posible obtener orina - glucosuria. En los restantes (No 20 y $25)$, tuvieron caracteres normales.

Las curvas anomales correspondieron a grados severos de anemia. a cifras de glucemia en ayunas inferiores a los normales y precedieron por pocos dias 


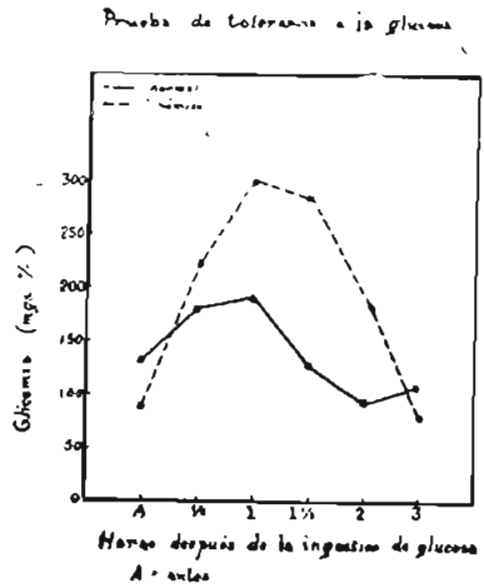

FIGURA No 4

Perro No 23.-Curva de tipo diabético (..) obtenida durante la etapa de anemia severa. Los exámenes complementarios el dia de la segunda prueba dieron los siguientes resultadoss eritrocitos 950.000 por $\mathrm{mm}^{3}$, leucocitos 20.800 por $\mathbf{m m}^{3}$, reticulocitos $14 \%$, eritrocitos parasitados $11 \%$, reacción indirecta de van den

$$
\text { Berg }++ \text {. }
$$

a la muerte del animal. En uno de estos casos (No 23, figura 4), la reacción indirecta de van den Berg fué positiva ++ , haciéndose dos dias más tarde la directa.

Las curvas de tolerancia normales (figura 5). correspondieron a numeraciones globulares superiores a 1.5 mills. por $\mathrm{mm}^{3}$; la glicemia en ayunas era normal y la reacción de van den Berg negativa. Los dos casos remitieron pocos

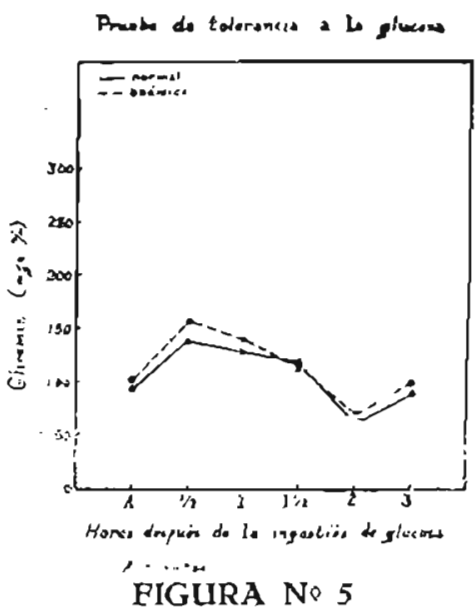

Perro No 25.-Curva de tipo normal (-.) obtenida durante el proceso anémico. Los exámenes complementarios el día de la segunda prueba dieron lo ssiguientes resultados: eritrocitos 1.77 mills por $\mathrm{mm}^{3}$, leucocitos 32.200 por $\mathrm{mm}$, reticulocitos $10 \%$, exitrocitos parasitados $5 \%$. Reacción indirecta de van den Berg negativa. 
dias después, alcanzando la curación aparente. Otra prueba realizada en uno de estos, durante la remisión sanguinea fué también normal.

Teniendo en cuenta que varios autores (36) han demostrado que el ayuno - restricción de carbohidratos en la dieta, tanto en el hombre como en los animales, puede determinar una curva de tolerancia alterada en el sentido diabético. nuestros casos fueron sometidos a una alimentación balanceada, uniforme, previvamente durante el desarrollo del proceso bartonellósico. ejerciêndose además un estricto control en la ingestión de ella.

No nos sería posible descartar la participación de una deficiencia de vitamina $B_{1}$ (causada por el proceso infeccioso-anémico) en la anormalidad constatada en las curvas de tolerancia, como ha sido demostrada por Pachman (37). estudiando la tolerancia a la administración de glucosa en ratas alimentadas con dieta deficiente en vitamina $B_{1}$.

Por último, otro factor que podría intervenir en la génesis del empobrecimiento del hígado en glucógeno, sería la reducción en la cantidad de alimentos ingeridos, causada por el estado de anorexia que a veces se presenta al final del proceso. En la mayor parte de nuestros casos hemos mantenido un severo control sobre la alimentación, y así hemos podido observar sindromes hipoglucémicos mortales en las primeras horas de la mañana (No 17 y 24 , figura 2), habiendo ingerido su ración alimenticia casi íntegramente el día anterior.

La escasez de glucógeno constatada en el hígado del perro con infección a Bartonella, de acuerdo con el concepto sostenido a partir de las experiencias de Whipple y Davis (38), que esta sustancia protege al parénquima hepático contra los tóxicos químicos y bacterianos y ayuda a su regeneración (28), desempeñaría un rol importante en la génesis de los focos de necrosis que han sido señalados en estos animales. Que en el área necrosada existió anteriormente a la muerte de las células una escasez de glucógeno, lo expresaria aquel halo de infiltración grasosa que la circunda - señalado por Weiss (9) - aceptando que esta última alteración no es sino un fenómeno compensador de la primera (28). En la Bartonellosis humana es probable que también ocurra esto; lo sugiere el halo grasoso que igualmente exhiben los focos necróticos.

Recientemente, Messinger y Hawkins (39), estudiando la influencia de la calidad de la dieta sobre la lesión del hígado producida por la arsfenamina, han encontrato que, si bien la dieta a base de carbohidratos da protección al hígado, la dieta proteínica es más beneficiosa, siendo las grasas perju. 
diciales en todos los casos. Este papel protector de las proteínas ha sido también constatado por Goldschmidt, Vars y Ravdin (40) y por Miller y Whipple (41) en ratas y perros sometidos a anestesia clorofórmica respectivamente. La lesión anatómica del hígado, producida por el cloroformo, se acentúa tanto como las proteínas se agotan en el hígado y tejidos del cuerpo. Según estudios de Miller (42), el aminoácido metionina y en menor grado la cistina, evitan la necrosis ciorofórmica en perros con reservas proteínicas agotadas.

En vista de la importancia de las proteínas, Miller y Whipple piensan que la acción benéfica de los carbohidratos se debería al ahorro de proteínas que el organismo hace cuando éstos son aportados en mayores cantidades que las habituales. En nuestro caso, es probable que el gran déficit de glucógeno en el hígado del perro bartoneliósico exprese también una disminución de las reservas proteínicas, sabiéndose que al agotarse los depósitos hidrocarbonados, el metabolismo proteico se acentúa, hecho demostrado en la inanición, fiebre, etc.

\section{Conclusiones.}

Se ha estudiado la glucemia en ayunas en sujetos normales, enfermos carriónicos y perros infestados con Bartonella canis. En estos últimos se ha realizado además dosajes de glucógeno hepático y pruebas de tolerancia a la glucosa. Los resultados obtenidos permiten las siguientes conclusiones:

1. - Una hiperglucemia de grado moderado puede ser constatada, con alguna frecuencia, en la fase hemática de la Enfermedad de Carrión.

2. - Esta alteración de la glucemia se presenta también en la Bartonellosis experimental del perro, durante la fase de anemia intensa.

3.-Una hipoglucemia, a veces de grado severo y responsable probablemente de la muerte del animal, acompaña con frecuencia a la etapa terminal de la Bartonellosis grave del perro, sucediendo a la hiperglucemia cuando ambos trastornos se presentan en el mismo caso. 
4. - La hiperglucemia constatada tanto en el enfermo carriónico como en el perro bartonellósico, puede ser explicada por una exagerada glucogenolisis hepática, estimulada por el estado infeccioso y quizás también por la anoxia anémica.

5.-Un agotamiento de las reservas de glucógeno en el hígado - producido principalmente por un mayor consumo de glucosa y una disminución de la capacidad del mismo para fijar aquella sustancia - y una probable inhibición de la neoglucogenia, serían los factores responsables del estado hipoglucémico que se presenta en la etapa terminal de la Bartonellosis grave del perro.

\section{BIBLIOGRAFIA}

(1).-Mann F. C. y Magath T. B. - Arch. Lnt. Med., 30, 73, 1922

(2).-Boliman J. L., Mann F. C. y Magath T. B. - Am. J. Physiol, 74, 238, 1925.

(3) .- Soskin S.. Essex H. E., Herrick J. F. y Mann F. C. - Am. J. Physiol., 124. $558,1938$.

(4).- Soskin S. - Endocrínology, 26, 297, 1940.

(5).--Freund H. A. - Ann. Lnt. Med., 10. 1144.1937.

(6). - Conn J. W. Newburgh L. H., Johnston M. W. y Sheldon J. M. Arch. Int. Med., 62, 765, 1938.

(7).-Coller F. A. y Troost F. L. - Ann. Surg., 90, 781. 1929.

(8) .- Strong R. P.. Tyzzer E. E., Sellards A. A., Brues C. T. y Gastiaburú J. C. : Report of last Expedition to South America, Harvard School of Tropical Medicine, Cambridge, Mass.. 1915.

(9).-Weiss P. y Pons J. - Act. Méd. Per., 4, No 6. 1938.

(10).-Pons J. y Urteaga O. - Acts. Acad. Cien. Ex. Fis. y Nat. de Lima, 2, 95, 1939.

(11).-Munch O. - A. J. Med. Sc., 191, 388, 1936.

(12). - Johnson A. - Acta Med. Scand., 3, 1939, 1922, (citado por 29).

(13) . - Kageura N. - J. Biochem. Japan, 1. 333, 1922, (citado por 22).

(14).-Gittler A. O. y St. George A. V. - J. A. M. A., 71, 2133, 1918. (citado por 29).

(15).-Burn J. H. : Biological Standardization, London, 1937.

(16) . -Folin O. - J. Biol. Chem., 86, 173, 1930.

(17).- Sorangyi M. - J. Biol. Chem., 86, 655, 1930.

(18) - - San Martin A. - An. Pac. Cien. Méd, 23, No 2, 1940.

(19).-Becher E. y asociados : Tratado de Fisiología Patológica Especial, Barcelona, 1936.

(20) - - Kirstein M. D. y Bromberg L. - J. Lab. and Clin. Med., 25, 7, 1939-40.

(21).-Gellhorn E. y Packer A. - Proc. Soc. Exper. Biol. and Med., 42, 475. 1939. 
(22) ---Hurtado A.. Pons J. y Merino C. : La Anemia de la Enfermedad de Carrión, Lima, 1938.

(23). - Soskin S. - Am. J. Physiol., 81, 382. 1927, (citado por 4).

(24).-Houssay B. A. y Foglia B. G. - Comp. rend. Soc. Soc. de Biol., 123, 824, 1936.

(25). - Sahyun M. - J. Biol. Chem., 93, 227, 1931.

(26) .- Sandberg M., Perla D. y Marmorston Gottesman J. - Exper. Med., 81, 57, 1933.

(27).-Coller F. A. y Jackson H. C. - J. A. M. A., 112. 128, 1939.

(28).-Rosenthal F. : Enfermedades del Higado y Vias Biliares, Buenos Aires. 1939.

(29).-Mc Master Ph. D. y Drury D. R. - J. Exper. Med., 49, 745, 1929.

(30) . -Conn J. W. - J. A. M. A., 115, 1669. 1940.

(31) .-Lucia S. P. y Aggeler P. M. - Am. J. Med. Sc., 201, 326, 1941.

(32).-Yzume S. y Lewis H. B. - J. Biol. Chem, 71, 51. 1926. (citado por 6).

(33).-Soskin S., Allweiss M. D. y Cohn D. J. - Am. J. Physiol., 109, 155. 1934.

(34). - Soskin S. y Allweiss M. D. - Am. J. Physiol., 110, 4. 1934.

(35) . - Citado por Coller F. A. y Jackson H. C., (30).

(36).-Conn J. W. - Am. I. Med. Sc., 199, 555, 1940.

(37). -Pachman D. I. - Aro. J. Physiol., 133, 43, 1941.

(38).-Davis N. C. y Whipple G. H. - Arch. Int. Med., 23. 612. 1919, (citado por 39).

(39) --Messinger W. J. y Hawkins W. B. - Am. I. Med. Sc., 199. 216.1940.

(40) - -Miller L. L. y Whipple G. H. - Am. J. Med. Sc., 199, 204, 1940.

(41). -Miller L. L. - Am. J. Med. Sc., 200, 739, 1940.

\section{SUMMARY}

The author studied the glycemia, prior to food being taken. in normal subjects, in patients suffering from Oroya Fever. and in dogs infected with Bartonella canis.

1.-A moderate hyperglycemia, can be proved to exist, with certain frequency, in the hematic stage of Carrion's disease.

2.- These changes in the glycemia, are also present, in the experimental dog Bartonelosis, during the stage of intense anaemia.

3.-An occasionally severe hypoglycemia, probably causing the animals death, often accompanies the terminal stage of serious cases of dog Bartonelosis, and follows the hyperglycemia whenever these two phenomena occur in one and the same case.

4.- The hyperglycemia proved to exist both in subjects suffering from Carrion's disease, and in dogs seized with Bartonelosis, can be explained as being due to an exagerated hepatic glycogenoly- 
sis, stimulated by the infectious state and also possibly by the anaemic anoxia.

5.- The exhaustion of the glycogene reserve of the liver, caused mainly by an over-consumption of glucose, and by the reduced capacity of the liver to fix this substance, and the probable inhibition of the neoglycogenolysis, would be the factors causing the hypoglycemic condition occurring in serious cases of dog Bartonelosis.

\section{RESUME}

On a etudié la glycémie à jeun chez des sujets normaux, des individus atteints de la maladie de Carrion et chez des chiens infectés par la Bartonella canis.

1.-Une hyperglycémie modérée peut être constatée, avec une certaine fréquence dans la phase hématique de la Maladie de Carrion.

2.-Cette altération de la glycémie se présente aussi dans la Bartonellose expérimentale du chien, pendant la phase d'ánemie intense.

3.-Une hypoglycémie, parfois sévére, et probablement responsable de la mort de l'animal, accompagne fréquemment l'étape terminale de la Bartonellose grave du chien. succédant à l'hyperglycémie quand ces deux phénoménes se présentent dans le même cas.

4.-L'hyperglycémie constatée, tant chez le sujet atteint de maladie de Carrion que chez le chien atteint de Bartonellose, peut être expliquée par une glycogénolyse hépatique exagérée, stimulée par l'état infectieux et peut-être aussi par l'anoxie anémique.

5. - Un épuisement des réserves de glycogéne dans le foie produit principalement par une plus grande consommation de glucose et par une diminution de la capacité du foie pour fixer cette substance - et une inhibition probable de la néoglycogénése. seraient les facteurs responsables de la l'état hypoglycémique qui se présente dans l'étape terminale de la Bartonellose grave du chien. 